\title{
Racial Differences in the Effectiveness of Internet-Delivered Mental Health Care
}

\author{
Charles R. Jonassaint, PhD, MHS ${ }^{1,2}$, Bea Herbeck Belnap, Dr Biol Hum ${ }^{7,3}$, Yan Huang, PhD ${ }^{4,5}$, \\ Jordan F. Karp, MD 5,6 , Kaleab Z. Abebe, PhD , and Bruce L. Rollman, MD, MPH
}

ICenter for Behavioral Health and Smart Technology, University of Pittsburgh, Pittsburgh, PA, USA; ${ }^{2}$ School of Medicine, University of Pittsburgh,
Pittsburgh, PA, USA; ${ }^{3}$ Department of Psychosomatic Medicine and Psychotherapy, University of Göttingen Medical Center, Göttingen, Germany;
${ }^{4}$ Center for Research on Health Care Data Center, University of Pittsburgh, Pittsburgh, PA, USA; ${ }^{5}$ Department of Psychiatry, University of Pittsburgh
School of Medicine, Pittsburgh, PA, USA; ${ }^{6}$ Geriatric Research, Education, and Clinical Center at VA Pittsburgh Health System, Pittsburgh, PA, USA.

BACKGROUND: Computerized cognitive behavioral therapy (cCBT) can improve mental health outcomes in White populations; however, it is unknown whether racial and ethnic minority populations receive clinical benefits from cCBT.

OBJECTIVE: To study race differences in the impact of cCBT use on mental health outcomes among White and African American primary care patients.

DESIGN: Secondary analysis of a three-arm randomized controlled clinical trial.

PARTICIPANTS: Primary care physicians (PCPs) referred 2,884 patients aged 18-75; 954 met eligibility criteria (including elevated mood and/or anxiety symptoms indicated as a score $\geq 10$ on Patient Health Questionnaire or Generalized Anxiety Disorder scale); 704 were randomized in 3:3:1 ratio to receive either (1) the cCBT program (cCBT-only), (2) cCBT plus access to an Internet Support Group (cCBT+ISG), or (3) their PCP's usual care (UC). After exclusions, this study analyzed 689 patients: 590 receiving cCBT, in the combined cCBT-only and cCBT+ISG groups (91 African American, 499 White), and 99 receiving UC (22 African American, 77 White).

INTERVENTION(S): We used the Beating the Blues cCBT program that consisted of eight 50-min Internet-delivered interactive sessions and "homework" assignments to

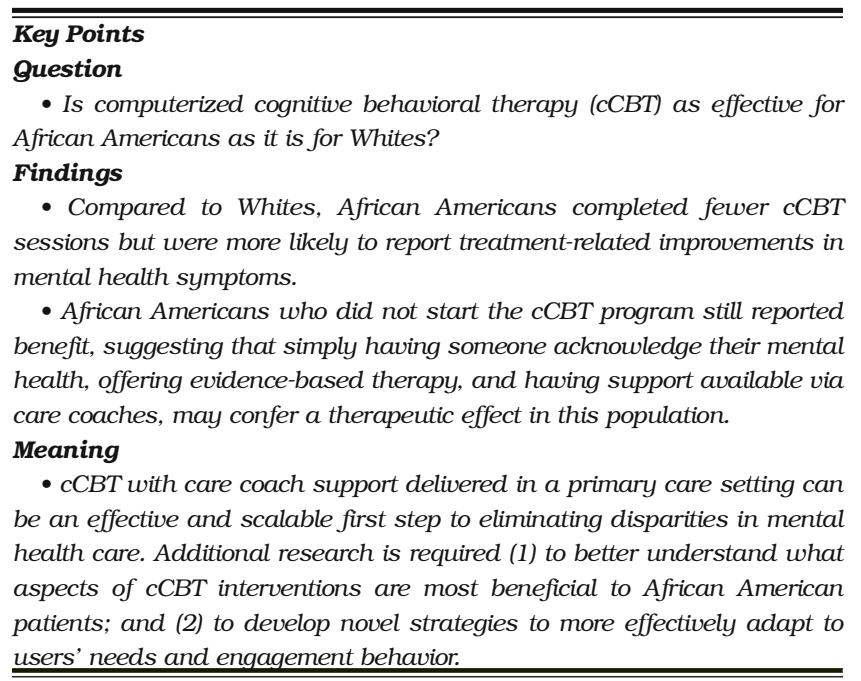

Received January 14, 2019

Revised July 17, 2019

Accepted October 8, 2019

Published online November 19, 2019 complete between weekly sessions. College graduatelevel care coaches provided six months of remote support. MAIN MEASURE(S): After prior analyses demonstrated no effect of the ISG program, we combined the cCBT-only and cCBT+ISG groups (cCBT) to compare to UC at 6-month follow-up. Controlling for sociodemographic factors, baseline symptoms, and treatment arm, we examined race differences for impact of cCBT versus UC on the mental health-related quality-of-life (Short-form 12 Health Survey), and Patient-Reported Outcomes Measurement Information System (PROMIS) anxiety, and depression.

RESULTS: Compared to UC, cCBT had no effect on quality of life $(d=0.10 ; p=0.40)$, depression $(d=-0.19 ; p=$ $0.10)$, or anxiety $(d=-0.16 ; p=0.18)$ for Whites. However, for African American patients, cCBT was associated with significant 6-month decrease in depression $(d=-0.47, p<$ $0.01)$ and anxiety scores $(d=-0.54, p<0.01)$.

CONCLUSIONS: cCBT may be an efficient and scalable first step to eliminating disparities in mental health care. TRIAL REGISTRATION: Clinicaltrials.gov Identifier: NCT01482806. https://www.clinicaltrials.gov/ct2 / show/NCT01482806?term=rollman\&rank=4

KEY WORDS: anxiety; cognitive behavioral therapy; depression; eHealth; healthcare disparities, ; mental health, ; minority health.

J Gen Intern Med 35(2):490-7

DOI: $10.1007 / \mathrm{s} 11606-019-05542-1$

(c) Society of General Internal Medicine 2019

\section{INTRODUCTION}

Depression and anxiety are common conditions in primary care and well-recognized causes of disability. ${ }^{1,2}$ Despite the high prevalence of mental health disorders in the primary care setting, patients often have difficulty accessing evidence-based treatments. This is particularly true for African Americans, ${ }^{3}$ who are at an increased risk for mood- and anxiety-related disorders becoming chronic. ${ }^{4}$ Even patients who do have access to mental health specialty care may choose to seek treatment for psychological distress from their primary care physician (PCP), an often less optimal level of treatment. ${ }^{5,6}$ However, African Americans' wariness toward mental health treatment, and 
lack of perceived need, may make them less likely to pursue any form of treatment, even through their PCP. ${ }^{6}$

As access to the Internet has become ubiquitous, online strategies for mental health treatment have emerged as an alternative to in-person psychotherapy. Internet-delivered computerized cognitive behavioral therapy (cCBT) has been proven effective at reducing depression and anxiety ${ }^{7,8}$; however, few cCBT trials have included non-White participants ${ }^{9}$ and examined racial/ethnic differences in cCBT outcomes. Little is known about how underrepresented minorities engage with $\mathrm{cCBT}$ and whether they benefit from this treatment modality. Two studies with over $50 \%$ of participants being African American found cCBT to be a helpful treatment for substance abuse among adults, ${ }^{10,11}$ and two pediatric studies examined cCBT as a treatment for mental health disorders, depression, ${ }^{12}$ and anxiety ${ }^{13}$ in cohorts that included nonWhite participants. Still, none of these studies examined racial differences in engagement or treatment effectiveness on outcomes. As a first step to closing this gap in the literature, our group examined self-reported outcomes for African American and White primary care patients receiving $\mathrm{cCBT}$ for mood and anxiety and found that the African American patients reported a greater 6-month decrease in depression and anxiety symptoms compared to Whites. ${ }^{14}$ However, these data were blinded, did not include a usual care comparison, and were self-reported outcomes. The next step, therefore, and the aim of the current study is to examine racial differences in mental health outcomes using blinded assessor ratings and a usual care group comparison in a large randomized clinical trial of online collaborative care for mood and anxiety disorders.

\section{METHODS}

This study is a secondary analysis of the NIMH-funded Online Treatment for Mood and Anxiety Disorders Trial (Online Treatment Trial; NCT01482806: PI Rollman), approved by the University of Pittsburgh's Institutional Review Board. This trial examined whether a cCBT program embedded in a primary care setting was effective in improving health-related quality of life and mood and anxiety symptoms, either alone or in combination with access to a moderated Internet Support Group. More detailed methods for the trial are available in the report of the study's primary outcomes. ${ }^{15}$

Primary care physicians (PCPs) from 26 offices in Pittsburgh, PA, a mid-sized Eastern city in the USA, all connected through an electronic medical record system, were notified of patients' possible trial eligibility via an automated electronic message. The PCP then referred interested anxious and/or depressed patients aged 18 to 75 , with reliable access to both the Internet and telephone, to the Online Treatment Trial. Study assessors contacted referred patients via telephone. Study consent forms were mailed to those who scored $\geq 10$ on either the Patient Health Questionnaire (PHQ-9) ${ }^{16}$ or the Generalized Anxiety Disorder scale (GAD-7), ${ }^{17}$ signifying at least a moderate symptom level, and who met all other eligibility criteria (e.g., no psychotic disorder; medically stable, not currently in mental health treatment). Upon receipt of the signed consent form, the assessors called the patients again and administered the baseline assessment, which included sociodemographics, use of pharmacotherapy, and the 12-item short-form health survey (SF-12) to determine health-related quality of life (HRQoL) ${ }^{18}$, fixed-length Patient-Reported Outcomes Measurement Information System (PROMIS) measures to assess mood and anxiety symptoms and pain interference $^{19}$, and Primary Care Evaluation of Mental Disorders (PRIME-MD) to provide an anxiety and mood disorder diagnosis. $^{20}$ The SF-12 has a mental health composite score (MCS) and physical health composite score (PCS). At 3 and 6 months, blinded assessors called each participant to repeat the baseline assessment battery.

\section{Randomization}

Protocol-eligible patients were randomized in a 3:3:1 ratio to (1) 24/7 patient access to a cCBT program provided under the guidance of a care coach (cCBT-only), (2) cCBT plus access to a moderated Internet Support Group (cCBT+ISG), or (3) their PCP's usual care (UC). Randomization was stratified by practice size and age group using randomly permuted block assignments. $^{21}$

\section{Computerized Cognitive Behavioral Therapy}

Patients randomized to either of the two intervention groups had Web access to the cCBT program Beating the Blues $(B t B)$, a proven-effective program ${ }^{22,23}$ that employs simple and easily understood text, audio, and audiovisual clips to maintain patient interest. The full program consists of eight 50-minlong interactive sessions and "homework" assignments to complete between weekly sessions.

\section{Care Coach Support}

College graduates with mental health research experience served as care coaches, and each supported participants exclusively in the cCBT treatment arms for 6 months to monitor patients' progress, provide support, and encourage continued engagement with the $\mathrm{CCBT}$ program. The preferred method of care coach contact was e-mail. If patients reported a worsening of symptoms and had not responded to e-mail messages, the care coach e-mailed or telephoned them. If a patient did not start or complete a session in more than 2 weeks, the care coach would e-mail them to encourage them to continue with the program. They also contacted patients if their symptoms did not improve; in cases of more complex psychosocial issues, or suicidal ideation; or to resolve technical problems. Care coaches reviewed their patient panel with a clinical specialty team consisting of a psychiatrist, psychologist, and general practitioner during weekly team supervision meetings. The team made recommendations including general lifestyle 
adjustments, and antidepressant/anxiolytic pharmacotherapy based on patients' treatment preferences and response to cCBT, as well as referrals to mental health specialists when they did not improve. ${ }^{15}$ We recorded the number of e-mails, telephone calls, and total contacts to each participant.

\section{Statistical Analyses}

We combined participants randomized to cCBT-only and cCBT+ISG into a single group (cCBT arm), as they both had access to the cCBT program and the study reporting the primary trial outcomes found no overall effect of the ISG program and no race differences in the effect of ISG. ${ }^{15}$ We compared sociodemographic factors and baseline symptoms between patients who self-identified as White or African American. Additionally, we compared the same characteristics between the cCBT and UC arms, stratified by self-identified race. All categorical variables were described using sample proportions and compared using a chi-square test; continuous variables were described using means and standard deviations and compared using two-sample $t$ tests.

In order to address our primary objective, we assessed whether race (White vs. African American) moderated the effect of cCBT (versus UC) on outcomes: SF-12 MCS, PROMIS anxiety, and PROMIS depression. Using linear mixed models, each of the outcomes was modeled as a function of study arm (cCBT vs. UC), time, race (African American vs White), and all 2- and 3-way interactions. Additionally, we adjusted for age group and site size as the randomization was stratified on these factors. Of interest was the significance of the 3-way interaction term, which would denote heterogeneity of the treatment effect across race groups. These analyses were hypothesis driven and based on our previously published findings. ${ }^{14}$

As a secondary analysis, we performed intensity-adjusted analyses in order to assess "how much" of the cCBT intervention resulted in improvements on outcomes. This was operationalized by fitting the same linear mixed models mentioned above but after replacing the study arm covariate with the number of cCBT sessions (ranging from 0 to 8). We ran similar models for number of care coach contacts and cCBT messages sent from the care coach to the user. Additionally, we assessed whether the relationship between number of cCBT sessions, contacts, messages, and associated outcomes differed between Whites and African Americans.

Estimates and 95\% confidence intervals for between-group differences and effect sizes were calculated based on mixed model contrasts. All significance tests were based on a type I error of 5\% with no adjustments for multiplicity. Statistical analyses were conducted in SAS ${ }^{\circledR} 9.4$ (Cary, NC).

\section{RESULTS}

Of 2,884 patients referred by PCPs, 954 met eligibility criteria, of whom 704 consented to the trial and were randomized (603
cCBT, $101 \mathrm{UC}$; Fig. 1). We excluded 15 participants who identified their race/ethnicity as other than White or African American/Caribbean Black (from here on referred to as African American) due to small race/ethnicity group numbers. The final sample of 689 included 590 patients assigned to cCBT (91 African American, 499 White) and 99 to the UC group (22 African American, 77 White).

Race and treatment group differences across baseline characteristics are shown in Table 1. At baseline, compared to Whites, the African American group was younger, included fewer males, was less likely to be married and employed, and reported lower social support. Those in the African American group were more likely to report major depression and lower physical quality of life. Additionally, African Americans reported slightly higher depressive symptoms on the PHQ-9 screen.

The mixed models comparing cCBT versus UC among Whites showed no effect of cCBT on 6-month change in SF$12 \operatorname{MCS}(d=0.10 ; p=0.40)$ or PROMIS-Depression and PROMIS-Anxiety scores $(d=-0.19 ; p=0.10 ; d=-0.16 ; p=$ 0.18; Table 2). For African Americans, cCBT was associated with greater improvement in SF-12 MCS $(d=0.25, p=0.25)$, although this difference was not statistically significant. However, African Americans in the cCBT group reported a significantly greater decrease in PROMIS-Depression $(d=-0.47, p$ $<0.01)$ and PROMIS-Anxiety scores $(d=-0.54, p<0.01)$ compared to those in the UC group (Table 3).

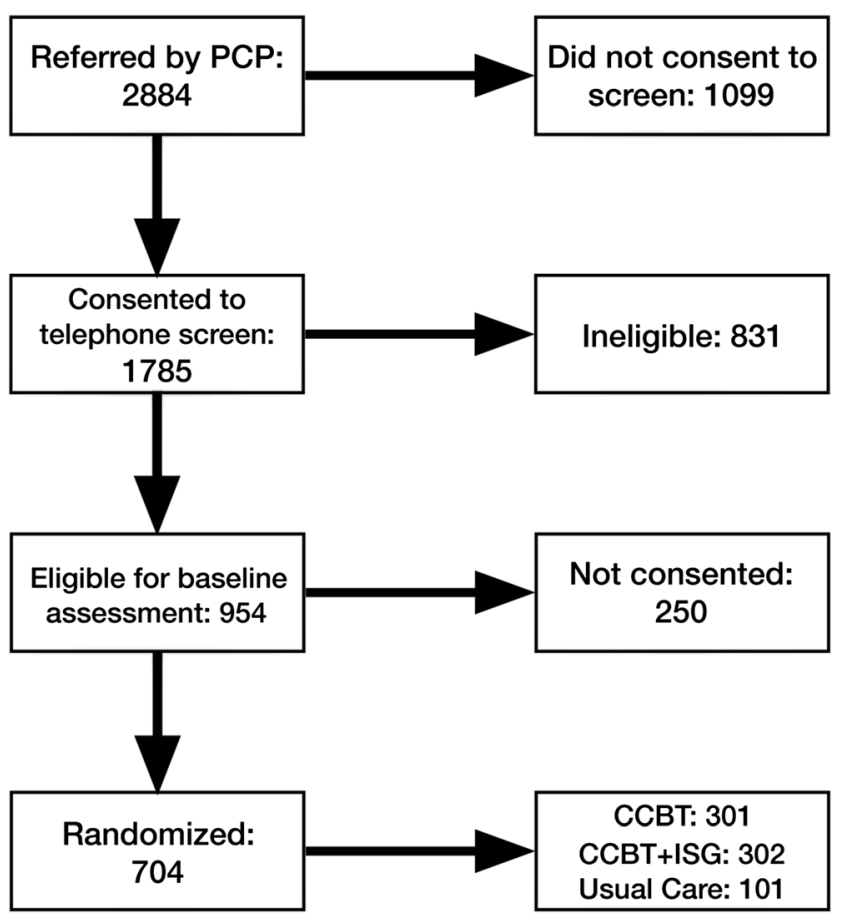

Figure 1 Consort diagram for Online Treatment for Mood and Anxiety Disorders Trial examining the effectiveness of a computerized cognitive behavioral therapy (cCBT) program embedded in a collaborative care approach for primary care patients with depression and anxiety. 
Table 1 Baseline Sociodemographic, Clinical Characteristics, and Engagement in Computerized Cognitive Behavioral Therapy Program (cCBT) for African American and White Race Primary Care Patients

\begin{tabular}{|c|c|c|c|c|c|c|c|}
\hline & \multicolumn{3}{|c|}{ African American } & \multicolumn{3}{|l|}{ White } & \multirow{2}{*}{$\begin{array}{l}\text { All African } \\
\text { American } \\
\text { vs Whites } \\
p \text { value }\end{array}$} \\
\hline & cCBT $(n=91)$ & $\mathrm{UC}(n=22)$ & $p$ value & cCBT $(n=499)$ & $\mathrm{UC}(n=77)$ & $p$ value & \\
\hline Age, mean (SD) & 40 (14) & $39.59(12.12)$ & 0.92 & $43.59(14.20)$ & $42(15)$ & 0.47 & 0.01 \\
\hline Female, \% $(N)$ & $87(79)$ & $91(20)$ & 0.60 & $78(390)$ & $77.9(60)$ & 0.96 & 0.02 \\
\hline HS degree or higher, $\%(N)$ & $77(70)$ & $86(19)$ & 0.33 & $83(414)$ & $83.12(64)$ & 0.97 & 0.28 \\
\hline Marital group, $\%(N)$ & & & 0.94 & & & 0.06 & $<0.01$ \\
\hline Single & $62.64(57)$ & $59.09(13)$ & NA & 35.87 (179) & $45.45(35)$ & NA & NA \\
\hline Married & $17.58(16)$ & $18.18(4)$ & & $44.89(224)$ & $45.45(35)$ & & \\
\hline Sep/div/widowed & $19.78(18)$ & $22.73(5)$ & & $19.24(96)$ & $9.09(7)$ & & \\
\hline Living alone, \% $(N)$ & 21 (19) & $9(2)$ & 0.36 & $18(92)$ & $10(8)$ & 0.08 & 0.75 \\
\hline Social support, mean (SD) & $61.07(18.52)$ & $65.09(14.56)$ & 0.34 & $67.49(16.04)$ & $70.47(13.35)$ & 0.08 & $<0.01$ \\
\hline Employed, \% $(N)$ & $57.14(52)$ & $68.18(15)$ & 0.34 & $71.74(358)$ & $71.43(55)$ & 0.95 & 0.009 \\
\hline Dx of PRIME-MD $, \S, \%(N)$ & & & 0.55 & & & 0.02 & 0.02 \\
\hline Depression only & $14.44(13)$ & $18.18(4)$ & NA & $18.84(94)$ & $9.09(7)$ & NA & NA \\
\hline Anxiety only & $2.22(2)$ & $4.55(1)$ & & $8.22(41)$ & $15.58(12)$ & & \\
\hline Both depression and anxiety & $83.33(75)$ & $77.27(17)$ & & $69.14(345)$ & $70.13(54)$ & & \\
\hline PHQ-9*, mean (SD) & $14.44(4.52)$ & $14.09(4.48)$ & 0.75 & $13.12(4.96)$ & $12.86(5.11)$ & 0.67 & 0.01 \\
\hline GAD $-7^{\dagger}$, mean (SD) & $13.16(4.15)$ & $12.55(4.45)$ & 0.54 & $12.78(4.42)$ & $13.75(4.14)$ & 0.07 & 0.76 \\
\hline $\begin{array}{l}\text { PROMIS Depression, } \\
T \text {-score mean (SD) }\end{array}$ & $63.32(6.80)$ & $61.07(5.89)$ & 0.16 & $62.15(6.00)$ & $61.57(6.60)$ & 0.44 & 0.20 \\
\hline $\begin{array}{l}\text { PROMIS Anxiety, } T \text {-score } \\
\text { mean (SD) }\end{array}$ & $66.69(5.86)$ & $64.44(5.83)$ & 0.11 & $65.78(5.98)$ & $65.73(5.78)$ & 0.94 & 0.43 \\
\hline SF-12 MCS, mean (SD) & $31.04(10.01)$ & $31.18(9.37)$ & 0.95 & $31.54(8.68)$ & $31.05(9.42)$ & 0.65 & 0.66 \\
\hline SF-12 PCS, mean (SD) & $47.03(13.14)$ & $49.20(16.87)$ & 0.51 & $51.52(12.03)$ & $53.32(11.21)$ & 0.22 & $<0.01$ \\
\hline Access to ISG, $\%(N)$ & $58.24(53)$ & NA & NA & $48.5(242)$ & NA & NA & 0.34 \\
\hline
\end{tabular}

*PHQ-9 = Patient Health Questionnaire 9-item Scale, assessor-administered

${ }^{\top} G A D-7=$ Generalized Anxiety Disorder 7-item Scale, assessor-administered

PHQ-9 and GAD-7 scores were assessed by research assistants, who were blinded to patient randomization status, over the telephone at baseline. The baseline assessor-administered PHQ-9 scores were highly correlated with self-entered PHQ-9 scores at session 1 ( $r=0.53 ; p<0.01)$. Race was selfreported. Primary Care Evaluation of Mental Disorders (PRIME-MD) was used to evaluate depression and anxiety diagnosis. All categorical variable $p$ values are based on a chi-square test. All continuous variable $p$ values are based on a t test or rank sum test for 2-group comparisons, and a KruskalWallis test or analysis of variance for 3-group comparisons. Adjusted models controlled for age, gender, education, PHQ-9, and baseline pharmacotherapy use

${ }^{7}$ Depression is defined as major depressive disorder (MDD) or dysthymia

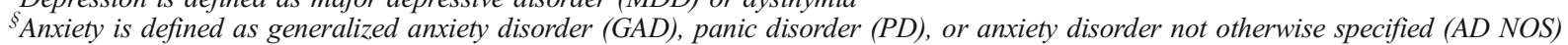

The three-way interaction testing whether cCBT has a differential benefit by race showed a marginal effect for PROMIS-Anxiety only ( $p=0.05)$, suggesting that compared to Whites, African Americans reported a greater benefit of the cCBT program on anxiety symptoms (African Americans: $d=$ $-0.54, p<0.01$; Whites: $d=-0.16, p=0.18)$. There was no significant subgroup effect of race on SF-12 MCS $(p=0.54)$ or PROMIS-Depression $(p=0.11)$.

We tested the association between the number of Beating the Blues (BtB) sessions completed and mental health outcomes. Whites completed more BtB sessions on average $(5.5, \mathrm{SD}=2.7)$ than African Americans $(4.7, \mathrm{SD}=$ 2.7; $p=0.03) .{ }^{14}$ For Whites, the number of BtB sessions completed was associated with 6-month improvements on all measures (Table 4); however, for African Americans, more sessions only conferred greater benefit on the PROMIS-Anxiety score $(p=0.014)$ (Table 3$)$. There was a significant race difference in the effect of number of sessions for SF12 MCS $(p=0.04)$. For Whites, only those who completed 4 or more BtB sessions reported greater improvements in SF12 compared to controls (Table 4), whereas all African Americans in the cCBT arm reported improvements in SF12, even if they completed zero BtB sessions (Table 4).
Examining number of care coach contacts, the African American patients who completed zero BtB sessions received more care coach contacts $($ mean $=11.19)$ than Whites who completed zero BtB sessions (mean $=8.30$ ). However, number of care coach contacts did not explain race differences in the effect of treatment or number of BtB sessions on outcomes (data not shown).

\section{DISCUSSION}

This study found that compared to usual care, cCBT conferred improvements in anxiety for African American but not for White primary care patients. Further, for the African American patients, cCBT was associated with 6-month improvements in depression and mental health-related quality of life. The more positive mental health impact of cCBT for African Americans as compared to Whites cannot be explained by number of Beating the Blues (BtB) sessions completed or frequency of care coach support.

Several systematic reviews show cCBT has a positive effect on mental health outcomes for Whites. ${ }^{8,24-26}$ Surprisingly, however, the current trial failed to show a robust cCBT effect for White patients despite cCBT having mental health benefits 
Table 2 Results of Mixed Models Comparing 6-Month Outcomes for White Primary Care Patients Randomized to Computerized Cognitive Behavioral Therapy (cCBT) Program Versus Usual Care (UC)

\begin{tabular}{|c|c|c|c|c|}
\hline White patients $(n=576)$ & cCBT $\quad(N=499)$ & $(N=77)$ & Between-group difference (95\% CI) & Cohen's $d(95 \% \mathrm{CI})$ \\
\hline \multicolumn{5}{|l|}{ MCS } \\
\hline Baseline & $31.94(0.49)$ & $31.44(1.23)$ & $1.17(-1.57,3.92) p$ value $=0.4015$ & $0.10(-0.13,0.32)$ \\
\hline 6 month & $44.20(0.52)$ & $42.53(1.28)$ & & \\
\hline Delta 6 month & $12.26(0.52)$ & $11.09(1.30)$ & & \\
\hline \multicolumn{5}{|l|}{ PROMIS-Anxiety } \\
\hline Baseline & $65.75(0.38)$ & $65.69(0.96)$ & $-1.40(-3.44,0.64) p$ value $=0.178$ & $-0.16(-0.40,0.07)$ \\
\hline 6 month & $56.98(0.41)$ & $58.31(1.00)$ & & \\
\hline Delta 6 month & $-8.77(0.39)$ & $-7.37(0.96)$ & & \\
\hline \multicolumn{5}{|l|}{ PROMIS-Depression } \\
\hline Baseline & $62.19(0.38)$ & $61.59(0.94)$ & $-1.55(-3.41,0.31) p$ value $=0.102$ & $-0.19(-0.43,0.04)$ \\
\hline 6 month & $53.50(0.40)$ & $54.46(0.97)$ & & \\
\hline Delta 6 month & $-8.68(0.35)$ & $-7.14(0.88)$ & & \\
\hline
\end{tabular}

for African Americans. These paradoxical racial differences for the cCBT effect may in part be due to the unexpectedly positive effect of usual care among White patients; in fact, White usual care patients reported 6-month improvements in mental health-related quality of life that was similar to White patients who completed 4 sessions of BtB. In contrast, African American patients in usual care reported less improvement in their mental health symptoms, whereas African Americans with access to cCBT reported larger improvements in symptoms even without completing any session of the BtB program or engaging with treatment through a care coach. Thus, from these preliminary data, it appears that African Americans in primary care may receive benefit in mental health symptoms simply by being offered and having available an intervention with support.

Racial disparities in access to and quality of mental health treatment may also partly explain the current findings showing a treatment response for African Americans but not Whites. For many African Americans in the study, this program may have been their first exposure to mental health screening and treatment. Indeed, more Whites were prescribed psychopharmacological interventions for mental health than African Americans (90\% vs. $76 \%$, respectively; $p<0.01) .{ }^{14}$ Unfortunately, this study did not collect data on race differences in history of mental health screening, frequency of prior discussions with the PCP regarding mental health, treatment referrals, treatment availability, or treatment uptake among primary care practices. Thus, it is difficult to interpret the potentially differential impact of usual care on mental health for Whites and African Americans. Further, it may be that Whites in the usual care arm, once identified as having depression, were more likely to seek out treatment on their own compared to African Americans, who are traditionally not likely to seek out treatment for depression/anxiety but instead attempt to manage it on their own. ${ }^{27,28}$ For many of the African American patients in this study, due to health care access and comfort level with mental health, the BtB program may have been their only real and perceived option for treatment, whereas the White patients in the study may have sought out or had mental health resources already in place.

Our previous report found that African Americans enrolled in cCBT were less likely to start the program (25\% of African Americans vs. $13 \%$ of Whites did not log in once) and completed fewer sessions than whites in the program (median 4.7 vs. 5.5). ${ }^{14}$ Despite completing fewer sessions, the African American patients still reported greater benefit from cCBT compared to Whites. Based on prior data from the current trial showing a dose-dependent response curve, in which the

Table 3 Results of Mixed Models Comparing 6-month Outcomes for African American Primary Care Patients Randomized to Computerized Cognitive Behavioral Therapy (cCBT) Program Versus Usual Care (UC)

\begin{tabular}{lllll}
\hline \hline $\begin{array}{l}\text { African American } \\
\text { Patients (n= 113) }\end{array}$ & cCBT (N = 91) & UC (N = 22) & Between-Group Difference (95\% CI) & Cohen's d (95\% CI) \\
\hline MCS & & & & \\
Baseline & $31.68(1.09)$ & $31.73(2.21)$ & $3.01(-2.10,8.13) p$ value $=0.248$ & $0.25(-0.13,0.64)$ \\
6 month & $43.81(1.12)$ & $40.84(2.21)$ & & $-0.54(-0.92,-0.16)$ \\
Delta 6 month & $12.13(1.18)$ & $9.11(2.33)$ & & \\
PROMIS-Anxiety & $66.47(0.85)$ & $64.20(1.72)$ & $-5.65(-9.44,-1.85) p$ value $=0.0036$ & \\
Baseline & $56.01(0.87)$ & $59.39(1.72)$ & & $-0.47(-0.85,-0.09)$ \\
6 month & $-10.46(0.87)$ & $-4.81(1.72)$ & & \\
Delta 6 month & $63.36(0.83)$ & $61.04(1.68)$ & $-4.75(-8.20,-1.31) p$ value $=0.0069$ & \\
PROMIS-Depression & $53.93(0.85)$ & $56.37(1.68)$ & & \\
Baseline & $-9.43(0.79)$ & $-4.67(1.57)$ & & \\
6 month & Delta 6 month & & & \\
\hline
\end{tabular}

The $p$ value for 3-way interaction restricted to 6 months for MCS model is 0.5349, for PROMIS-Anxiety is 0.0536, and for PROMIS-Depression is 0.1084 
Table 4 Mean 6-Month Improvement for Usual Care, and as a Function of no. of Beating the Blues (BtB) Sessions completed Within the First 6 Months for White and African American Primary Care Patients

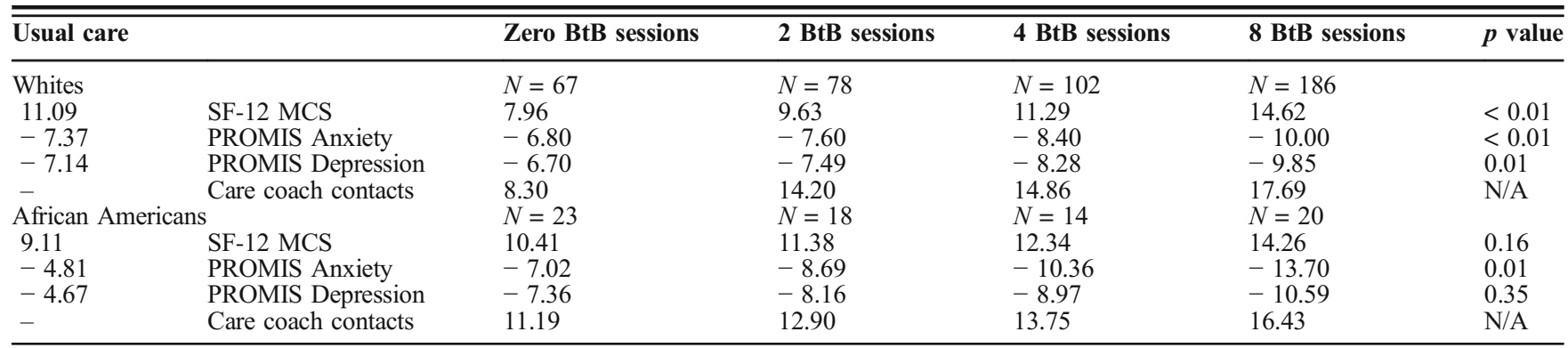

$p$ value is from the test statistic for testing the interaction between no. of sessions and time

outcomes improved as the number of completed sessions increased, we would expect that African Americans would have less benefit from cCBT; however, we show in the current analyses that number of sessions completed had no impact on outcomes in African Americans, whereas it did for Whites. In fact, those African Americans who had access to cCBT but did not start the program still reported marginally greater reduction in mental health symptoms as compared to the UC group. Thus, the CBT skills acquisition alone likely does not explain improvement in clinical symptoms reported by African American patients. Indeed, since patients formed a positive alliance with the program that is comparable to a face-to-face psychotherapies ${ }^{29}$, it is possible that African Americans had a strong belief that the treatment would work and their symptoms would improve. Therefore, even the opportunity to engage in a treatment they perceived as effective conferred benefit.

Computerized self-help programs are most effective when the treatment is professionally supported. ${ }^{24}$ Care coaches in the current study provided a high level of psychological support to participants and made on average 14 contacts with each participant. This is particularly critical given that participants' perceived absence of support has been noted as a primary reason for poor adherence and treatment drop-out. ${ }^{30}$ In a large-scale pragmatic trial comparing $\mathrm{cCBT}$ (Beating the Blues and MoodGYM) to usual care, ${ }^{31}$ the cCBT intervention showed no benefit over usual care in improving depressive symptoms, a result that was likely due to perceived inadequate level of support, ${ }^{30}$ and as a potential consequence, one quarter of the participants had dropped out of the study by 4 months.

Given the importance of close and regular clinical support, it is possible that even patients who did not acquire any new CBT skills benefited from receiving e-mails and calls from the study care coach. We hypothesized that care coach support would be particularly beneficial for African American study patients due to this group's reported lower level of social support as compared to Whites. However, we found no significant effect of care coach support contacts on mental health outcomes to help explain the differential effect of the cCBT intervention by race. Although not examined in the current study, patient-program alliance has been associated with cCBT engagement. ${ }^{29}$ Thus, as with face-to-face clinical encounters, future research may find that racial concordance or perceived personal similarity between the patient and the care coach is linked to improved outcomes. ${ }^{32}$

In addition to differences in treatment outcomes, this study also identified several sociodemographic disparities between African Americans and Whites that may be associated with mental health risk. African Americans in this study were less likely to be married or/and reported lower levels of social support compared to their White counterparts. These findings are not unique to this sample as studies have consistently found that African Americans have a lower socioeconomic status, ${ }^{33}$ are less likely to get married and more likely to get divorced ${ }^{34}$ and report smaller social networks and lower perceived social support. ${ }^{35}$ Although these factors did not impact the within group differences (cCBT vs UC) in outcomes observed in our primary analyses, all of these sociodemographic factors have been found to play a role in physical and mental health. ${ }^{33,36}$ Thus, future programs to address health disparities likely need to consider that impact of social determinants of poor health and not simply implementing treatments that focus on the readily addressable, surface-level, mental health concerns.

Limitations. Two main features of the current study may have limited its generalizability. This study only included patients from primary care practices in one geographic location. Second, only patients with Internet access and a desktop or laptop computer at home were eligible to participate. Thus, we may have excluded several lowerincome patients who may have benefited from the program but did not have home Internet access. Despite these restrictions, the sample was relatively racially and socioeconomically diverse, with over $18 \%$ of participants being African American and 1 out of 5 participants having less than a high school education.

Another major limitation of the study is our lack of details regarding current or history of psychological treatment and qualitative data regarding patient perceptions of the cCBT program, data that may help elucidate the racial differences in treatment response. Finally, this study lacked the qualitative interviews necessary to fully understand the non-specific components of the CCBT intervention arm that African American patients found beneficial. 


\section{CONCLUSIONS}

Several systematic reviews have demonstrated the benefit of cCBT programs. However, because prior trials included almost exclusively White participants, we lack data on whether these evidence-based cCBT programs are as effective for minority populations. Interestingly, the current trial data from a primary care setting hint that African American primary care patients may derive more benefit from an Internet-delivered cCBT program than their White counterparts, who traditionally have had better access to mental health care.

The widespread deployment of cCBT into primary care could become an efficient and replicable first step to eliminating disparities in mental health care. However, two things are needed for this to become a reality: first, additional research is required in order to understand what aspects of the cCBT intervention are most beneficial to African American patients; second, novel strategies are needed for increasing engagement, such as programs that are customized for different cultures and that will adapt to the user and their behavior. ${ }^{29,37}$

Corresponding Author: Charles R. Jonassaint, PhD, MHS; School of Medicine University of Pittsburgh, Pittsburgh, PA, USA (e-mail: cjonassaint@pitt.edu).

Funding Information This project was supported by grant numbers R01MH093501 (PI: Rollman) from the National Institute of Mental Health, K23HL135396 (PI: Jonassaint) from the National Heart Lung and Blood Institute, and K12HSO22989 from the Agency for Healthcare Research and Quality.

\section{Compliance with Ethical Standards:}

Conflict of Interest: The authors declare they do not have a conflict of interest.

\section{REFERENCES}

1. Kessler RC, Demler O, Frank RG, et al. Prevalence and treatment of mental disorders, 1990 to 2003. N Engl J Med 2005;352(24):2515-2523.

2. Mathers CD, Loncar D. Projections of global mortality and burden of disease from 2002 to 2030. PLoS Med 2006;3(11):e442.

3. Stockdale SE, Lagomasino IT, Siddique J, McGuire T, Miranda $\mathbf{J}$. Racial and ethnic disparities in detection and treatment of depression and anxiety among psychiatric and primary health care visits, 19952005. Med Care 2008;46(7):668-677.

4. Himle JA, Baser RE, Taylor RJ, Campbell RD, Jackson JS. Anxiety disorders among African Americans, blacks of Caribbean descent, and non-Hispanic whites in the United States. J Anxiety Disord 2009;23(5):578-590.

5. Williams DR, Gonzalez HM, Neighbors H, et al. Prevalence and distribution of major depressive disorder in African Americans, Caribbean blacks, and non-Hispanic whites: results from the National Survey of American Life. Arch Gen Psychiatry 2007;64(3):305-315.

6. Neighbors HW, Caldwell C, Williams DR, et al. Race, ethnicity, and the use of services for mental disorders: results from the National Survey of American Life. Arch Gen Psychiatry 2007;64(4):485-494.

7. Adelman CB, Panza KE, Bartley CA, Bontempo A, Bloch MH. A metaanalysis of computerized cognitive-behavioral therapy for the treatment of DSM-5 anxiety disorders. J Clin Psychiatry 2014;75(7):e695-704.

8. Andrews G, Cuijpers P, Craske MG, McEvoy P, Titov N. Computer therapy for the anxiety and depressive disorders is effective, acceptable and practical health care: a meta-analysis. PLoS One 2010;5(10):e13196.
9. Knowles SE, Toms G, Sanders C, et al. Qualitative meta-synthesis of user experience of computerised therapy for depression and anxiety. PLoS One 2014;9(1):e84323.

10. Kiluk BD, Nich C, Carroll KM. Relationship of cognitive function and the acquisition of coping skills in computer assisted treatment for substance use disorders. Drug Alcohol Depend 2011;114(2-3):169-176.

11. Budney AJ, Stanger C, Tilford JM, et al. Computer-assisted behavioral therapy and contingency management for cannabis use disorder. Psychol Addict Behav 2015;29(3):501-511.

12. Iloabachie $\mathbf{C}$, Wells $\mathbf{C}$, Goodwin B, et al. Adolescent and parent experiences with a primary care/Internet-based depression prevention intervention (CATCH-IT). Gen Hosp Psychiatry 2011;33(6):543-555.

13. Khanna MS, Kendall PC. Computer-assisted cognitive behavioral therapy for child anxiety: results of a randomized clinical trial. J Consult Clin Psychol 2010;78(5):737-745.

14. Jonassaint CR, Gibbs P, Belnap BH, Karp JF, Abebe KK, Rollman BL. Engagement and outcomes for a computerised cognitive-behavioural therapy intervention for anxiety and depression in African Americans. BJPsych Open 2017;3(1):1-5.

15. Rollman BL, Herbeck Belnap B, Abebe KZ, et al. Effectiveness of Online Collaborative Care for Treating Mood and Anxiety Disorders in Primary Care: A Randomized Clinical Trial. JAMA Psychiatry 2018;75(1):56-64.

16. Kroenke K, Spitzer RL, Williams JBW. The PHQ-9: Validity of a brief depression severity measure. J Gen Intern Med 2001;16(9):606-613.

17. Spitzer RL, Kroenke K, Williams JBW, Löwe B. A Brief Measure for Assessing Generalized Anxiety Disorder: The GAD-7. Arch Intern Med 2006; 166(10): 1092-1097.

18. Ware J, Jr., Kosinski M, Keller SD. A 12-Item Short-Form Health Survey: construction of scales and preliminary tests of reliability and validity. Med Care 1996;34(3):220-233.

19. Choi SW, Reise SP, Pilkonis PA, Hays RD, Cella D. Efficiency of static and computer adaptive short forms compared to full-length measures of depressive symptoms. Qual Life Res 2010;19(1):125-136.

20. Spitzer RL, Williams JB, Kroenke K, et al. Utility of a new procedure for diagnosing mental disorders in primary care. The PRIME-MD 1000 study. JAMA. 1994;272(22):1749-1756.

21. Matts JP, Lachin JM. Properties of permuted-block randomization in clinical trials. Control Clin Trials 1988;9(4):327-344.

22. Proudfoot J, Ryden C, Everitt B, et al. Clinical efficacy of computerised cognitive-behavioural therapy for anxiety and depression in primary care: randomised controlled trial. Br J Psychiatry 2004; 185:46-54.

23. Kaltenthaler E, Brazier J, De Nigris E, et al. Computerised cognitive behaviour therapy for depression and anxiety update: a systematic review and economic evaluation. Health Technol Assess 2006; 10(33):iii, xi-xiv, 1168.

24. Andersson G, Cuijpers P. Internet-based and other computerized psychological treatments for adult depression: a meta-analysis. Cogn Behav Ther 2009;38(4):196-205.

25. Newby JM, Twomey C, Yuan Li SS, Andrews G. Transdiagnostic computerised cognitive behavioural therapy for depression and anxiety: A systematic review and meta-analysis. J Affect Disord 2016; 199:30-41.

26. Richards D, Richardson T. Computer-based psychological treatments for depression: a systematic review and meta-analysis. Clin Psychol Rev 2012;32(4):329-342.

27. Kohn-Wood L, Hooper L. Cultural competency, culturally tailored care, and the primary care setting: Possible solutions to reduce racial/ethnic disparities in mental health care. J Ment Health Couns 2014;36(2):173188.

28. Cook BL, Zuvekas SH, Carson N, Wayne GF, Vesper A, McGuire TG. Assessing racial/ethnic disparities in treatment across episodes of mental health care. Health Serv Res 2014;49(1):206-229.

29. Cavanagh K, Herbeck Belnap B, Rothenberger SD, Abebe KZ, Rollman BL. My care manager, my computer therapy and me: The relationship triangle in computerized cognitive behavioural therapy. Internet Interv 2018;11:11-19.

30. Knowles SE, Lovell K, Bower P, Gilbody S, Littlewood E, Lester H. Patient experience of computerised therapy for depression in primary care. BMJ Open 2015;5(11):e008581.

31. Gilbody S, Littlewood E, Hewitt $\mathbf{C}$, et al. Computerised cognitive behaviour therapy (cCBT) as treatment for depression in primary care (REEACT trial): large scale pragmatic randomised controlled trial. BMJ. 2015;351:h5627.

32. Street RL, Jr., O'Malley KJ, Cooper LA, Haidet P. Understanding concordance in patient-physician relationships: personal and ethnic dimensions of shared identity. Ann Fam Med 2008;6(3):198-205. 
33. Williams DR, Priest N, Anderson NB. Understanding associations among race, socioeconomic status, and health: Patterns and prospects. Health Psychol 2016;35(4):407-411.

34. Raley RK, Sweeney MM, Wondra D. The Growing Racial and Ethnic Divide in U.S. Marriage Patterns. Futur Child 2015;25(2):89-109.

35. Flores M, Ruiz JM, Goans C, et al. Racial-ethnic differences in social networks and perceived support: Measurement considerations and implications for disparities research. Cult Divers Ethn Minor Psychol 2019.

36. Ruiz JM, Hamann HA, Mehl MR, O'Connor M-F. The Hispanic health paradox: From epidemiological phenomenon to contribution opportunities for psychological science. Group Process Intergroup Relat 2016;19(4):462-476.

37. Griner D, Smith TB. Culturally adapted mental health intervention: A meta-analytic review. Psychotherapy (Chic) 2006;43(4):531-548.

Publisher's Note Springer Nature remains neutral with regard to jurisdictional claims in published maps and institutional affiliations. 